RAE-IC, Revista de la Asociación Española de Investigación de la Comunicación vol. 9, núm. 17 (2022), 25-50 ISSN 2341-2690

Recibido el 21 de febrero de 2022 DOI: https://doi.org/10.24137/raeic.9.17.2 Aceptado el 21 de febrero de 2022

\title{
Reciclajes seriales en la era de los servicios de vídeo bajo demanda
} Serial recycling in times of video-on-demand services

\section{Cascajosa-Virino, Concepción}

Universidad Carlos III de Madrid (UC3M)

ccvirino@hum.uc3m.es

\section{Forma de citar este artículo:}

Cascajosa-Virino, C. (2022). Reciclajes seriales en la era de los servicios de vídeo bajo demanda. RAE-IC, Revista de la Asociación Española de Investigación de la Comunicación, 9(17), 25-50. https://doi.org/10.24137/raeic.9.17.2

\section{Resumen:}

El reciclaje de materiales narrativos ha sido uno desde los inicios de la serialidad televisiva un mecanismo habitual para la generación de nuevos contenidos, utilizando referentes tanto externos como internos. Sin embargo, en los últimos años ha experimentado un importante impulso ligado a la llegada de los servicios de vídeo bajo demanda y la consecuente explosión cuantitativa de la producción. Las series que adaptan o expanden películas y series precedentes han alcanzado un peso específico, especialmente en los servicios de los grupos mediáticos tradicionales (legacy media). Esta investigación tiene como objetivo analizar las características de esta tendencia de producción en alza y de la manera en la que está perfilando los contenidos. Para ello, se tomarán de referencia tanto los principales servicios de vídeo demanda transnacionales 
(Amazon Prime Vídeo, Apple+, Disney+, HBO Max, Hulu, Netflix, Paramount+ y Peacock) como españoles (Atresplayer Premium, Movistar+). Los resultados permiten valorar la importancia del reciclaje narrativo en la producción audiovisual contemporánea, su función en la construcción de marcas y su relación con la nostalgia.

Palabras clave: producción audiovisual, reciclaje narrativo, series de ficción, servicios de vídeo bajo demanda

\section{Abstract:}

The recycling of narrative materials has been a common mechanism for the generation of new content since the beginnings of television seriality, using both external and internal references. However, in recent years it has experienced a significant boost due to the arrival of video-on-demand services and the consequent quantitative production boom. Fiction series that adapt or expand previous films and series have achieved a specific relevance particularly in the services of traditional media groups (legacy media). This article analyses the characteristics of this growing production trend and the way in which it is shaping content. To do so, the main transnational (Amazon Prime Vídeo, Apple+, Disney+, HBO Max, Hulu, Netflix, Paramount+ y Peacock) and Spanish video-ondemand services (Atresplayer Premium, Movistar+) will be analysed. The results help to understand the relevance of narrative recycling in contemporary audio-visual production, its role in brand building and its relationship with nostalgia.

Keywords: audio-visual production, narrative recycling, fiction series, video-on-demand services

\section{INTRODUCCIÓN}

El proceso de digitalización ha tenido importantes repercusiones en el sistema audiovisual contemporáneo, transformando las fórmulas de producción, distribución y consumo. Como señala Amanda Lotz:

La combinación de una variedad de tecnologías de distribución y una variedad de modelos de negocio permite un futuro televisivo más polifacético, que 
probablemente no reunirá audiencias masivas con determinados programas de la manera habitual hasta ahora. El futuro ofrece considerables oportunidades para los espectadores, los creadores y las industrias, aunque puedan ser muy diferentes del pasado (Lotz, 2021, p. 151. Traducción propia).

Uno de los cambios más significativos en el ecosistema audiovisual ha sido la llegada de los servicios de vídeo bajo demanda por suscripción (subscription video-on-demand services, SVOD), los más relevantes procedentes de Estados Unidos pero con ambición transnacional. Netflix, nacido en Estados Unidos en 1997, ha ocupado una posición de liderazgo en este proceso introduciendo importantes novedades en la distribución de contenido (como el estreno de temporadas completas el mismo día y la organización algorítmica del catálogo) con repercusiones en el consumo, como el binge-watching o visionado en atracones (Jenner, 2018).

Tras encontrar un modelo de negocio basado en un modelo de suscripción por tarifa plana, Netflix se concentró, especialmente a partir de 2013, en una estrategia paralela de expansión de la producción de contenido original e internacionalización: en 2021 estimó una inversión de 17 mil millones de dólares en contenido (Low, 2021) disponible para los 221,8 millones de subscriptores que tenía en todo el mundo (Spangler, 2022). La estela de Netflix fue seguida por otras empresas de base tecnológica como Amazon (con Amazon Prime Vídeo, en 2006) y Apple (con Apple TV+, en 2019), que han lanzado sus propios servicios de vídeo bajo demanda con producción original.

Durante la etapa de lanzamiento y expansión de estos servicios bajo demanda, los operadores tradicionales, tanto de la televisión generalista como del pago, mantuvieron estrategias de colaboración como una ventana adicional para rentabilizar su contenido, pero algunos, de manera paralela, se aventuraron a lanzar sus propios servicios. Uno de los ejemplos más relevantes fue Hulu, un proyecto conjunto creado por en 2007 por News Corporation y NBC Universal al que se sumó posteriormente The Walt Disney Company, y que integraba contenido de las tres empresas y originales disponibles tanto con publicidad como a través de modalidades de suscripción (Sanson y Steirer, 2019). Esta estrategia de colaboración empezó a modificarse tras constatar el crecimiento imparable de Netflix y la manera en la que la continuada erosión de la audiencia 
cuestionaba su propia viabilidad. Muchos de estos operadores, parte de conglomerados que ya integraban estudios cinematográficos y empresas editoriales, cambiaron su estrategia de licencia de contenidos para lanzar sus propios servicios.

En este periodo, bautizado en los medios de comunicación como "Streaming Wars" (Neira, 2020), ha visto la emergencia de servicios como Disney+ (2019), Peacock (2020), HBO Max (2020) y Paramount+ (2021, aunque ya estaba en funcionamiento como CBS All Access desde 2014). En el contexto español, Movistar+, la plataforma de televisión de pago resultado de la adquisición de Canal+ por Telefónica, ha ido orientado su contenido al consumo bajo demanda y en 2019 creó un servicio de suscripción de vídeo bajo demanda separado llamado Movistar+ Lite. Por su parte, los operadores de televisión generalista lanzaron ese mismo año sus propios servicios de suscripción, Mediaset con MiTele Plus y Atresmedia con Atresplayer Premium (Fernández, 2021).

La llegada de todos estos servicios de vídeo bajo demanda se ha materializado en un importante crecimiento de la inversión en contenido. El Financial Times estimó que los 8 principales grupos de comunicación de Estados Unidos iban a invertir 115 miles de millones de dólares en la producción de cine, series y programas en 2022 (Nicolaou y Grimes, 2021). En este contexto, las series de ficción se han convertido en uno de los contenidos predilectos, gracias a su capacidad para fidelizar audiencias y estimular un tipo de consumo intensivo. Como producto cultural, las series vivieron en la primera década del siglo XXI un proceso de revalorización debido al salto cualitativo de calidad en la televisión de pago (Cascajosa Virino, 2016). Sin embargo, el inicio de la producción de series por los servicios de vídeo bajo demanda tuvo consecuencias más cuantitativas. En 2015 el presidente del canal de cable FX, John Landgraf, acuñó el término "Peak TV" para referirse al crecimiento acelerado de la producción: el número de series de ficción pasó de 211 en 2009 a 371 en 2014 (Schneider, 2015). Los servicios de vídeo bajo demanda mantuvieron esta tendencia al alza: en 2021 el número llegó a 559 (White, 2022).

Este aceleramiento de la producción se fue replicando conforme estos servicios de vídeo demanda comenzaron, como parte de su internacionalización, a producir localmente. En 2020, Netflix ya se había convertido en el principal productor de series de ficción de Europa, por encima de ZDF, BBC y France TV, según la consultora Ampere Analysis 
(Dziadul, 2021). En mercados como el español, la producción de series de ficción también experimentó un fuerte incremento: de 26 títulos en 2014 a 50 en 2020 (Mateos-Pérez y Sirera-Blanco, 2021, p. 8). Según el cálculo del periódico El Español, entre Netflix, Amazon Vídeo, Movistar+ y Atresplayer Premium sumaron el 74\% de los 55 estrenos de series de ficción de 2021 (Morillo, 2021).

El crecimiento de la producción ha supuesto nuevas oportunidades para el ámbito de la creación, pero también se ha producido un impulso al reciclaje de materiales narrativos previos. En el contexto de la economía de la atención (Davenport y Beck, 2001), las adaptaciones y expansiones de narrativas previas han ido alcanzado mayor relevancia por una variedad de factores, como la eficacia demostrada, la necesidad de destacar en un mercado saturado de oferta y la potencialidad para la expansión franquiciada.

Estos programas de televisión, que derivan de otros textos mediáticos o reactivan una serie mucho tiempo después de su primera emisión, se basan principalmente en el conocimiento previo de la audiencia a través de la familiaridad con los nombres de marca. Suelen cortejar a las bases de fans y suelen estructurarse en torno a los vínculos sentimentales persistentes con los personajes de ficción y los "mundos narrativos" que habitan. En los últimos años, los títulos reconocibles han sido muy esperados por los espectadores, que parecen estar en constante alerta por el regreso de sus programas favoritos (Loock, 2017, pp. 301-302. Traducción propia).

En 2019 la consultora Ampere Analysis determinó las adaptaciones habían crecido de manera sostenible en los últimos años, hasta llegar a alcanzar el tercio de las nuevas series en la primavera de ese año. Además de novelas y libros (49\%), destacaban fórmulas de reciclaje narrativo audiovisual como el remake (12\%), el spin-off (7\%) y el reboot (4\%) (Bisson, 2019).

En las siguientes páginas se analizarán las características de esta tendencia en la producción de series que adaptan y expanden narrativas previas procedentes del propio medio audiovisual (cine y series) y su relevancia en las estrategias de producción original de las plataformas de vídeo bajo demanda, estableciendo un marco comparativo entre Estados Unidos y España. 


\section{MARCO TEÓRICO}

Esta investigación se basa en los ámbitos teóricos diferenciados. Por un lado, la bibliografía académica dedicada a la producción de contenido original por los servicios de vídeo bajo demanda. Por otro, la dedicada a los procesos de adaptación y expansión narrativa en la ficción audiovisual y, específicamente, en las series contemporáneas.

\subsection{LA PRODUCCIÓN DE LOS SERVICIOS DE VÍDEO BAJO DEMANDA}

La bibliografía académica dedicada a la irrupción de los servicios de vídeo bajo demanda en el ecosistema audiovisual ha mostrado un especial énfasis en Netflix debido a su popularidad internacional y liderazgo en la expansión de los nuevos consumos audiovisuales. Aunque una parte de esta bibliografía se ha centrado en aspectos relacionados con el consumo (Castro, Rigby, Cabral y Nisi, 2019), otra se ha ocupado de su marco regulatorio (Novak, 2016; García Leiva y Albornoz, 2021), su configuración industrial en el contexto de la emergencia de las plataformas (Lotz, 2017) y su expansión con ambición transnacional (Lobato, 2019; Lobato y Lotz, 2021).

Especialmente relevantes para esta investigación han sido los trabajos que se han ocupado de su producción original de series. Mientras la importancia de la producción original empieza a ser un aspecto de interés para analizar la construcción de marca de estos servicios (Palomba, 2022), el concepto se sigue usando de forma flexible dentro de los catálogos de estos servicios y especialmente en Netflix. De acuerdo con el analista Matthew Ball (2018), la compañía usa en su catálogo el término "Original” para referirse a hasta cuatro contenidos diferenciados: "Developed Originals" ("Originales desarrollados"), “Acquired Originals" (“Originales adquiridos”), "Co-Licensing" (“CoLicencia”) y “Licensed Originals" (“Originales licenciados”).

Como parte de un esfuerzo general en "redefinir las características de las series de televisión de éxito en el contexto de las plataformas transnacionales y las audiencias globales" (Wayne y Uribe Sandoval, 2021, p. 2), Netflix inició una intensa inversión en ficción fuera de las mediciones tradicionales de audiencia que pronto se extendió globalmente (lordache, Raats y Afilipoaie, 2021; Mastrini y Krakowiak, 2021). La producción original de los servicios de vídeo bajo demanda se presenta todavía como 
un fenómeno reciente, pero ya ha despertado una intensa atención crítica en aspectos relativos a la representación (Villarejo, 2016) y localización (Scarlata, Lobato y Cunningham, 2021).

\subsection{EL RECICLAJE DE MATERIALES NARRATIVOS EN LA FICCIÓN AUDIOVISUAL CONTEMPORÁNEA}

El patrón de reconocimiento que Derek Thompson (2017) identificaba como clave para la popularidad de los productos culturales y la transformación de la industria cultural ha llevado a una relevancia cada mayor de las fórmulas basadas en el reciclaje de materiales narrativos. En su fórmula más expandida, la franquicia, donde los materiales se extienden a través de diferentes medios, adaptándose a los cambios en las tendencias relativas a las audiencias y al marco tecnológico (Fleury, Hartzheim y Mamber, 2019, p. 1). Tal y como afirma Derek Johnson:

Especialmente en las últimas tres décadas, la franquicia ha puesto en tensión la consolidación conglomerada de la propiedad intelectual y el impulso para compartirla; la utilidad de los medios emergentes para las instituciones establecidas y la amenaza potencial de esos mismos medios; la expansión del mercado a través de la multiplicación y la contracción de los nichos de mercado; la racionalización económica y la imaginación afectiva de nuevos modelos para generar cultura (Johnson, 2013, pp. 6-7. Traducción propia).

Ello también ha convertido a los estudios sobre adaptación ha sido en las últimas décadas un fértil campo de trabajo en la bibliografía académica, particularmente los dedicados a la intersección entre cine y literatura (Leitch, 2008). Poco a poco también ha ido alcanzando relevancia otras fórmulas de reciclaje narrativo, especialmente aquellas articuladas sobre los intercambios entre cine y televisión (Cascajosa Virino, 2006). De manera gradual, se han ido incorporando nuevas categorías a las fórmulas de reciclaje, partiendo de las tradicionales continuaciones (secuelas y precuelas, esta última una continuación que se desarrolla antes de la narrativa original) y versiones (remakes). El concepto reboot, asociado al cómic antes de ser utilizado en cine y televisión, es una variedad del remake articulado sobre la noción de serialidad, en el sentido de reiniciar una serie de textos en lugar de reversionar un texto aislado, lo que 
lo relaciona con la franquicia (Gil, 2014). Por su parte, en el ámbito de las series se ha popularizado el concepto de revival para definir la reactivación de una narrativa serial que ha estado mucho tiempo fuera de producción manteniendo buena parte de sus elementos constitutivos previos (Loock, 2018).

Así han ido sumándose trabajos que han indagado sobre fórmulas de reciclaje narrativo cada más populares en el Hollywood contemporáneo y por extensión en las industrias del cine y la televisión globales. Ha sido el caso de la secuela (Jess-Cooke, 2009; Henderson, 2017), pero también de la versión o remake como categoría industrial, textual o crítica (Verevis, 2006). Rosewarne (2020) ha establecido una taxonomía que explica los principales fundamentos del remake como práctica industrial en Hollywood: el remake mejorado, el remake económico, el remake nostálgico, el remake americanizado, el remake creativo y el remake a la moda.

Por su parte, autores como Herbert (2018) han explorado cómo los creadores contemporáneos han utilizado las versiones o remakes para introducir comentarios sociales actualizados o elementos de diversidad no presentes en las encarnaciones anteriores. Pero la importancia del remake ha trascendido su función en la producción de Hollywood para alcanzar una relevancia global, permitiendo la reflexión sobre cuestiones como la emergencia de lo transnacional (Smith y Verevis, 2017) y las lógicas continentales (Cuelenaere, Willems y Joye, 2021) y nacionales (Fernández Labayen y Martín Morán, 2019).

De manera equivalente, el reciclaje de materiales narrativos ha ido ganando importancia en las estrategias de producción de series, y especialmente en los servicios de vídeo bajo demanda. La bibliografía académica ha prestado especial atención a fórmulas de reciclaje que han sido históricamente relevantes en el contexto de la televisión norteamericana, como la versión de series internacionales (Perkins y Verevis, 2019) y el spin-off, en donde un personaje secundario de una narrativa se convierte en protagonista de otra (Bellamy, McDonald y Walker, 1990). Por su parte, la emergencia de las versiones de series clásicas y revivals se ha basado en la construcción de un capital nostálgico (Lavigne, 2014, p. 1), algo que especialmente Netflix ha explotado con el 
propósito de apelar y retener a suscriptores con ayuda del conocimiento algorítmico de sus preferencias (Pallister, 2019, p. 3).

\section{METODOLOGÍA}

La investigación tiene como objetivo examinar el reciclaje de materiales narrativos audiovisuales en las series original de imagen real de los servicios de vídeo bajo demanda. Sobre la base previa de una aproximación cuantitativa, se realizará un análisis de las características de estas producciones y su relación con estrategias de los diferentes servicios y los grupos mediáticos de los que dependen.

Para realizar este análisis se adopta una perspectiva comparada. Por un lado, se han tenido en cuenta las series de ficción producidas en Estados Unidos, de donde proceden los principales servicios transnacionales y el crecimiento de la producción ha sido más temprano (Netflix estrenó su primera serie en 2013) e intenso (hasta superar los 500 títulos en 2021). Las plataformas analizadas en este apartado han sido ocho: tres de compañías de base tecnológica (Amazon Prime Vídeo, Apple TV+ y Netflix) y cinco de grupos de comunicación tradicionales, los denominados legacy media (Disney+, HBO Max, Hulu, Paramount+ y Peacock). Aunque se tiene en consideración que servicios como Netflix y Amazon Prime Vídeo llevan casi una década produciendo series originales, se considera oportuna la comparación con servicios más recientes para comprobar la evolución de tendencias.

Por otro lado, se han tenido en cuenta las series producidas en España desde la llegada de los servicios de vídeo bajo demanda. En este caso, se han analizado de forma separada los servicios nacionales (Movistar+ y Atresplayer Premium) y los servicios transnacionales con producción original en España (Amazon Prime Vídeo, Disney+, HBO y Netflix). Aunque el periodo estudiado es relativamente corto (cinco años), se pueden extraer conclusiones relevantes.

Se ha creado una base de datos con la producción original desde el estreno de la primera serie de ficción original de cada servicio de vídeo bajo demanda hasta febrero de 2022. La base de datos se ha elaborado analizando como fuente primaria los catálogos de los propios servicios, que se han contrastado con fuentes secundarias como la IMDB. Solo 
se han incluido si cuentan con una película o serie como referente acreditado, aunque haya otra referencia complementaria (por ejemplo, una novela). La ficha de cada serie incluye el servicio de vídeo bajo demanda a cuyo catálogo pertenece, el periodo temporal de su estreno y finalización (si ya no tiene continuidad), el tipo de reciclaje narrativo y el material precedente. Se han establecido seis tipos de reciclaje narrativo en las series de ficción analizadas:

- Versión temporal: Versión de una serie o película del mismo país de origen.

- Versión espacial: Versión una serie o película de otro país.

- Continuación: Expansión de una película o serie, ya sea prospectiva o retrospectivamente.

- Revival: Continuación de una serie que mantiene sus elementos narrativos esenciales tras su cancelación.

- Reboot: Versión de una serie con diferencias significativas en sus elementos narrativos esenciales.

- Spin-off: Serie en la que uno o varios personajes secundarios de una película o serie previa alcanzan el estatus de protagonista.

Para esta definición se han tenido en cuenta que, más allá de sus características, algunas de estas categorías (como el spin-off, el revival y el reboot) existen como fórmulas específicas tanto de producción como de recepción crítica.

\section{RESULTADOS}

\subsection{EL RECICLAJE EN LA PRODUCCIÓN ORIGINAL DE LOS SERVICIOS DE VÍDEO BAJO} DEMANDA EN ESTADOS UNIDOS

La base de datos de series originales de los servicios de vídeo bajo demanda en Estados Unidos está formada por 62 series. La distribución por servicios es la siguientes: Netflix (20 títulos), Disney+ (11 títulos), CBS All Access / Paramount + (9 títulos), Peacock (6 títulos), Hulu (5 títulos), Amazon Prime Vídeo (4 títulos), HBO Max (4 títulos) y Apple TV+ (3 títulos). Por tipología, la distribución es la siguiente: continuación (20 títulos), versión espacial (13 títulos), versión temporal (11 títulos), spin-off (9 títulos), revival (6 títulos) y reboot (3 títulos). Respecto a los años de estreno, el desglose es el siguiente: 2013 (1 
título), 2015 (2 títulos), 2016 (2 títulos), 2017 (9 títulos), 2018 (7 títulos), 2019 (7 títulos), 2020 (8 títulos), 2021 (20 títulos) y 2022 (hasta febrero, 6 títulos). A continuación, se procede al desglose por servicio de vídeo bajo demanda.

\subsubsection{Netflix.}

Netflix es un servicio de vídeo bajo demanda por suscripción fundado en 1997 y con presencial global que estrena series de ficción originales desde 2013. En los últimos años, ha desarrollado un fuerte impulso a la producción de contenido, que se ha complementado con la apertura de nuevas líneas de negocio en el campo editorial y los videojuegos.

Netflix ha sido particularmente activa en las continuaciones (8 títulos), versiones temporales (4 títulos) y revivals ( 2 títulos), estableciendo una vinculación entre su producción original y el contenido adquirido, tanto películas como series de televisión.

Tabla 1. Reciclajes narrativos en la producción original de Netflix en EE.UU.

\begin{tabular}{|c|c|c|c|c|}
\hline Título & Servicio & Producción & Tipo & Material previo \\
\hline House of Cards & Netflix & $2013-2018$ & Versión espacial & $\begin{array}{c}\text { Serie House of Cards } \\
\text { (1990, Reino Unido) }\end{array}$ \\
\hline $\begin{array}{c}\text { Wet Hot } \\
\text { American } \\
\text { Summer: First } \\
\text { Day of Camp }\end{array}$ & Netflix & 2015 & Continuación & $\begin{array}{c}\text { Película Wet Hot } \\
\text { American Summer } \\
\text { (2001) }\end{array}$ \\
\hline $\begin{array}{c}\text { Gilmore Girls: A } \\
\text { Year in the Life }\end{array}$ & Netflix & 2016 & Revival & $\begin{array}{c}\text { Serie Gilmore Girls } \\
\text { (The WB/The CW: } \\
\text { 2000-2007) }\end{array}$ \\
\hline $\begin{array}{c}\text { Fuller House } \\
\text { Wet Hot } \\
\text { American } \\
\text { Summer: Ten } \\
\text { Years Later }\end{array}$ & Netflix & $2016-2020$ & Revival & $\begin{array}{c}\text { Serie Full House (ABC: } \\
\text { 1987-1995) }\end{array}$ \\
\hline $\begin{array}{c}\text { The Defenders } \\
\text { Netflix }\end{array}$ & Netflix & 2017 & Continuación & $\begin{array}{c}\text { Serie Wet Hot } \\
\text { American Summer: } \\
\text { First Day of Camp } \\
\text { (2015) }\end{array}$ \\
\hline $\begin{array}{c}\text { One Day at a } \\
\text { Time }\end{array}$ & Netflix & $2017-2019$ & Versión temporal & $\begin{array}{c}\text { Serie One Day at a } \\
\text { Time (CBS: 1975- } \\
\text { 1984) }\end{array}$ \\
\hline $\begin{array}{c}\text { She's Gotta } \\
\text { Have It }\end{array}$ & Netflix & $2017-2019$ & Versión temporal & $\begin{array}{c}\text { Película She's Gotta } \\
\text { Have It (1986) }\end{array}$ \\
\hline $\begin{array}{c}\text { Greenhouse } \\
\text { Academy }\end{array}$ & Netflix & $2017-2020$ & Versión espacial (2015- & $\begin{array}{c}\text { Serie Ha-Hamama } \\
\text { (2012-2016, Israel) }\end{array}$ \\
\hline $\begin{array}{c}\text { Dear White } \\
\text { People }\end{array}$ & Netflix & $2017-2021$ & Versión temporal & $\begin{array}{c}\text { Película Dear White } \\
\text { People (2014) }\end{array}$ \\
\hline
\end{tabular}




\begin{tabular}{|c|c|c|c|c|}
\hline Título & Servicio & Producción & Tipo & Material previo \\
\hline Maniac & Netflix & 2018 & Versión espacial & $\begin{array}{c}\text { Serie Maniac (2015, } \\
\text { Noruega) }\end{array}$ \\
\hline Seven Seconds & Netflix & 2018 & Versión espacial & $\begin{array}{c}\text { Película Maŭop } \\
\text { (2013, Rusia) }\end{array}$ \\
\hline Lost in Space & Netflix & 2018 & Versión espacial & $\begin{array}{c}\text { Serie Ha-Shoter Ha- } \\
\text { Tov (2015-2017, } \\
\text { Israel) }\end{array}$ \\
\hline $\begin{array}{c}\text { Narcos: Mexico } \\
\text { Black Summer }\end{array}$ & Netflix & $2018-2021$ & Continuación & $\begin{array}{c}\text { Serie Narcos (Netflix: } \\
\text { 2015-2017) }\end{array}$ \\
\hline $\begin{array}{c}\text { The Dark } \\
\text { Crystal: Age of }\end{array}$ & Netflix & 2019 & Continuación & $\begin{array}{c}\text { Serie Z Nation (SyFy: } \\
\text { 2014-2018) }\end{array}$ \\
\hline $\begin{array}{c}\text { Resistance } \\
\text { Ratched }\end{array}$ & Netflix & 2019 & Continuación & $\begin{array}{c}\text { Película The Dark } \\
\text { Crystal (1982) }\end{array}$ \\
\hline Cowboy Bebop & Netflix & $2020-$ & Continuación & $\begin{array}{c}\text { Película One Flew } \\
\text { Over the Cuckoo's } \\
\text { Nest (1975) }\end{array}$ \\
\hline $\begin{array}{c}\text { Vikings: Valhalla } \\
\text { Netflix }\end{array}$ & $2022-$ & Continuación & $\begin{array}{c}\text { Serie Vikings (History } \\
\text { Channel: 2013-2020) }\end{array}$ \\
\hline
\end{tabular}

Fuente: Elaboración propia

\subsubsection{Disney+}

Disney+ es un servicio de vídeo bajo demanda por suscripción lanzado por The Walt Disney Company en noviembre de 2019 y que alcanzó escala transnacional tras su llegada diferentes mercados en Europa, América y Asia. Disney+ incluye en su catálogo contenido procedente de las diferentes compañías del conglomerado, como Marvel, Disney, ABC, Star Wars y National Geographic, que se utilizan como etiquetas de organización de contenido.

Su producción de ficción original de series de ficción se ha articulado casi exclusivamente sobre la propiedad intelectual de estas compañías, especialmente a través de los spinoffs de Marvel (4 títulos) y Star Wars (2 títulos), y películas de Disney (3 títulos).

Tabla 2. Reciclajes narrativos en la producción original de Disney+ en EE.UU.

\begin{tabular}{|c|c|c|c|c|}
\hline Título & Servicio & Producción & Tipo & Material previo \\
\hline $\begin{array}{c}\text { The } \\
\text { Mandalorian }\end{array}$ & Disney+ & 2019- & Spin-off & $\begin{array}{c}\text { Franquicia } \\
\text { cinematográfica Star } \\
\text { Wars (1977-) }\end{array}$ \\
\hline $\begin{array}{c}\text { High School } \\
\text { Musical: The } \\
\text { Series }\end{array}$ & Disney+ & 2019- & Reboot & $\begin{array}{c}\text { Franquicia de } t v- \\
\text { movies High School } \\
\text { Musical (2006-2011) }\end{array}$ \\
\hline
\end{tabular}




\begin{tabular}{|c|c|c|c|c|}
\hline Título & Servicio & Producción & Tipo & Material previo \\
\hline The Right Stuff & Disney+ & 2020 & Versión temporal & $\begin{array}{c}\text { Película The Right } \\
\text { Stuff (1983) }\end{array}$ \\
\hline WandaVision & Disney+ & 2021 & Spin-off & $\begin{array}{c}\text { Franquicia } \\
\text { cinematográfica } \\
\text { Marvel (2008-) }\end{array}$ \\
\hline $\begin{array}{c}\text { The Falcon and } \\
\text { the Winter } \\
\text { Soldier }\end{array}$ & Disney+ & 2021 & Spin-off & $\begin{array}{c}\text { Franquicia } \\
\text { cinematográfica } \\
\text { Marvel (2008-) }\end{array}$ \\
\hline Loki & Disney+ & 2021 & Spin-off & $\begin{array}{c}\text { Franquicia } \\
\text { cinematográfica } \\
\text { Marvel (2008-) }\end{array}$ \\
\hline $\begin{array}{c}\text { Doogie } \\
\text { Kameāloha, } \\
\text { M.D. }\end{array}$ & Disney+ & 2021 & Versión temporal & $\begin{array}{c}\text { Serie Doogie Howser, } \\
\text { M.D. (ABC: 1989- } \\
\text { 1993) }\end{array}$ \\
\hline Hawkeye & Disney+ & 2021 & Spin-off & $\begin{array}{c}\text { Franquicia } \\
\text { cinematográfica } \\
\text { Marvel (2008-) }\end{array}$ \\
\hline $\begin{array}{c}\text { The Book of } \\
\text { Boba Fett }\end{array}$ & Disney+ & 2021 & Spin-off & $\begin{array}{c}\text { Franquicia } \\
\text { cinematográfica Star } \\
\text { Wars (1977-) }\end{array}$ \\
\hline Turner \& Hooch & Disney+ & 2021 & Continuación & $\begin{array}{c}\text { Película Turner \& } \\
\text { Hooch (1989) }\end{array}$ \\
\hline $\begin{array}{c}\text { The Mighty } \\
\text { Ducks: Game } \\
\text { Changers }\end{array}$ & Disney+ & 2021- & Continuación & $\begin{array}{c}\text { Franquicia } \\
\text { cinematográfica The } \\
\text { Mighty Ducks (1992- } \\
\text { 1996) }\end{array}$ \\
\hline
\end{tabular}

Fuente: Elaboración propia

\subsubsection{CBS All Access / Paramount+}

Paramount+ es un servicio de vídeo bajo demanda propiedad de Paramount Global, compañía conocida como ViacomCBS Inc. hasta comienzos del 2022. Se trata de un conglomerado que incluye la cadena generalista CBS, la cadena de cable Showtime y el estudio cinematográfico Paramount. El servicio fue lanzado como CBS All Access en 2014, y fue renombrado Paramount+ en 2021.

Durante sus primeros años de actividad, el servicio se utilizó como segunda ventana para los contenidos del grupo, y de manera gradual empezó a producir contenido original relacionado con series de la cadena CBS (como el spin-off The Good Fight y la continuación The Twilight Zone) y la franquicia de Paramount Star Trek (3 títulos). En su etapa más reciente, este es el ámbito en el que se mantienen sus originales (incluyendo a las cadenas de cable Paramount y Nickelodeon). 
Tabla 3. Reciclajes narrativos en la producción original de CBS All Access / Paramount+ en EE.UU.

\begin{tabular}{|c|c|c|c|c|}
\hline Título & Servicio & Producción & Tipo & Material previo \\
\hline The Good Fight & $\begin{array}{c}\text { CBS All Access / } \\
\text { Paramount + }\end{array}$ & 2017- & Spin-off & $\begin{array}{c}\text { Serie The Good Wife } \\
\text { (CBS: 2009-2016) }\end{array}$ \\
\hline No Activity & $\begin{array}{c}\text { CBS All Access / } \\
\text { Paramount + }\end{array}$ & 2017- & Versión espacial & $\begin{array}{c}\text { Serie No Activity } \\
\text { (2015-2018, } \\
\text { Australia) }\end{array}$ \\
\hline $\begin{array}{l}\text { Star Trek: } \\
\text { Discovery }\end{array}$ & $\begin{array}{c}\text { CBS All Access / } \\
\text { Paramount + }\end{array}$ & 2017- & Continuación & $\begin{array}{c}\text { Franquicia Star Trek } \\
(1966-)\end{array}$ \\
\hline $\begin{array}{c}\text { Star Trek: Short } \\
\text { Treks }\end{array}$ & $\begin{array}{c}\text { CBS All Access / } \\
\text { Paramount + }\end{array}$ & 2018-2020 & Continuación & $\begin{array}{c}\text { Franquicia Star Trek } \\
(1966-)\end{array}$ \\
\hline Tell Me a Story & $\begin{array}{c}\text { CBS All Access / } \\
\text { Paramount + }\end{array}$ & 2018-2020 & Versión espacial & $\begin{array}{l}\text { Serie Cuéntame un } \\
\text { cuento (2014, } \\
\text { España) }\end{array}$ \\
\hline $\begin{array}{c}\text { The Twilight } \\
\text { Zone }\end{array}$ & $\begin{array}{c}\text { CBS All Access / } \\
\text { Paramount + }\end{array}$ & 2019-2020 & Continuación & $\begin{array}{c}\text { Serie The Twilight } \\
\text { Zone (CBS: 1959- } \\
\text { 1964) }\end{array}$ \\
\hline Star Trek: Picard & $\begin{array}{c}\text { CBS All Access / } \\
\text { Paramount + }\end{array}$ & 2020- & Continuación & $\begin{array}{c}\text { Franquicia Star Trek } \\
(1966-)\end{array}$ \\
\hline 1883 & $\begin{array}{c}\text { CBS All Access / } \\
\text { Paramount + }\end{array}$ & 2021- & Continuación & $\begin{array}{c}\text { Serie Yellowstone } \\
\text { (Paramount Network: } \\
\text { 2018-) }\end{array}$ \\
\hline iCarly & $\begin{array}{c}\text { CBS All Access / } \\
\text { Paramount + }\end{array}$ & 2021- & Revival & $\begin{array}{c}\text { Serie iCarly } \\
\text { (Nickelodeon: 2007- } \\
\text { 2012) } \\
\end{array}$ \\
\hline
\end{tabular}

Fuente: Elaboración propia

\subsubsection{Peacock}

Peacock es un servicio de vídeo bajo demanda por suscripción propiedad de NBCUniversal. El servicio fue lanzado en los Estados Unidos el 15 de julio de 2020, y tiene como objetivo una expansión transnacional a través de acuerdos con otros operadores como Sky y Paramount Global. Propiedad del conglomerado Comcast, el grupo tiene cadenas generalistas como NBC y Telemundo, cadenas de cable como SyFy y estudios cinematográficos como Universal Pictures y DreamWorks Animation.

A pesar de llevar en funcionamiento poco tiempo, ha utilizado las fórmulas de reciclaje de forma significativa en su producción original de series originales que toman de referencia el catálogo de ficciones de NBC (5 títulos), destacando entre ellas el revival (2 títulos). 
Tabla 4. Reciclajes narrativos en la producción original de Peacock en EE.UU.

\begin{tabular}{|c|c|c|c|c|}
\hline $\begin{array}{c}\text { Título } \\
\begin{array}{c}\text { Saved by the } \\
\text { Bell }\end{array}\end{array}$ & Servicio & Producción & Tipo & $\begin{array}{c}\text { Material previo } \\
\text { Serie Saved by the } \\
\text { Bell (NBC: 1989-1993) }\end{array}$ \\
\hline $\begin{array}{c}\text { Days of Our } \\
\text { Lives: Beyond } \\
\text { Salem }\end{array}$ & Peacock & $2020-$ & Revival & $\begin{array}{c}\text { Serie Days of Our } \\
\text { Lives (NBC: 1965) }\end{array}$ \\
\hline $\begin{array}{c}\text { The Girl in the } \\
\text { Woods }\end{array}$ & Peacock & 2021 & Versión temporal & $\begin{array}{c}\text { Cortometrajes The } \\
\text { Door in the Woods } \\
\text { (2018) y The Girl in } \\
\text { the Woods (2020) }\end{array}$ \\
\hline Punky Brewster & Peacock & 2021 & Revival & $\begin{array}{c}\text { Serie Punky Brewster } \\
\text { (NBC/Sindicación: } \\
\text { 1984-1988) }\end{array}$ \\
\hline MacGruber & Peacock & $2021-$ & Continuación & $\begin{array}{c}\text { Sketch de Saturday } \\
\text { Night Live (NBC: } \\
\text { 2007-2010) / Película } \\
\text { MacGruber (2010) }\end{array}$ \\
\hline Bel-Air & Peacock & 2022- & Versión temporal & $\begin{array}{c}\text { Serie The Fresh Prince } \\
\text { of Bel-Air (NBC: 1990- } \\
\text { 1996) }\end{array}$ \\
\hline
\end{tabular}

Fuente: Elaboración propia

\subsubsection{Hulu}

Hulu es un servicio de vídeo bajo demanda con modalidades de suscripción y por publicidad que actualmente es propiedad de The Walt Disney Company, NBC Universal como socio minoritario. Lanzado en 2007, estrenó su primera serie original en 2011, y actualmente es un servicio complementario a Disney+ orientado a audiencias adultas.

El reciclaje de material audiovisual ha tenido una relevancia pequeña dentro de su abundante producción original con títulos que adaptaban películas (3 títulos). Sin embargo, en el periodo más reciente destacan las continuaciones de series de empresas del conglomerado, como FX y 20th Century Fox Television (2 títulos).

Tabla 5. Reciclajes narrativos en la producción original de Hulu en EE.UU.

\begin{tabular}{|c|c|c|c|c|}
\hline Título & Servicio & Producción & Tipo & Material previo \\
\hline $\begin{array}{c}\text { Four Weddings } \\
\text { and a Funeral }\end{array}$ & Hulu & 2019 & Versión espacial & $\begin{array}{c}\text { Película Four } \\
\text { Weddings and } a \\
\text { Funeral (1994) }\end{array}$ \\
\hline A Teacher & Hulu & 2020 & Versión temporal & $\begin{array}{c}\text { Película A Teacher } \\
\text { (2013) }\end{array}$ \\
\hline Love, Victor & Hulu & $2020-$ & Continuación & $\begin{array}{c}\text { Película Love Simon } \\
\text { (2018) }\end{array}$ \\
\hline $\begin{array}{c}\text { American Horror } \\
\text { Stories }\end{array}$ & Hulu & $2021-$ & Continuación & $\begin{array}{c}\text { Serie American } \\
\text { Horror Story (FX: } \\
\text { 2011-) }\end{array}$ \\
\hline
\end{tabular}




\begin{tabular}{|c|c|c|c|c|}
\hline Título & Servicio & Producción & Tipo & Material previo \\
\hline $\begin{array}{c}\text { How I Met Your } \\
\text { Father }\end{array}$ & Hulu & 2022- & Continuación & $\begin{array}{c}\text { Serie How I Met Your } \\
\text { Mother (CBS: 2005- } \\
\text { 2014) }\end{array}$ \\
\hline
\end{tabular}

Fuente: Elaboración propia

\subsubsection{Amazon Prime Video}

Amazon Prime Vídeo es un servicio de vídeo bajo demanda por suscripción propiedad de la tienda virtual Amazon.com. Lanzado en 2006, Amazon Prime Vídeo comenzó a estrenar series originales en 2013 de forma complementaria a la producción de películas y de otro tipo de contenido de entretenimiento.

Frente a su frecuente utilización de referentes literarios, el reciclaje de material audiovisual ha sido una fórmula marginal en Amazon Prime Vídeo. Únicamente destaca la utilización las versiones espaciales de series internacionales (3 títulos).

Tabla 6. Reciclajes narrativos en la producción original de Amazon Prime Vídeo en EE.UU.

\begin{tabular}{|c|c|c|c|c|}
\hline Título & Servicio & Producción & Tipo & Material previo \\
\hline Mad Dogs & $\begin{array}{c}\text { Amazon Prime } \\
\text { Vídeo }\end{array}$ & $2015-2016$ & Versión espacial & $\begin{array}{c}\text { Serie Mad Dogs } \\
\text { (2011-2013, Reino } \\
\text { Unido) }\end{array}$ \\
\hline Hanna & $\begin{array}{c}\text { Amazon Prime } \\
\text { Vídeo }\end{array}$ & $2019-2021$ & Versión temporal & Película Hanna (2011) \\
\hline Utopia & $\begin{array}{c}\text { Amazon Prime } \\
\text { Vídeo }\end{array}$ & 2020 & Versión espacial & $\begin{array}{c}\text { Serie Utopia (2013- } \\
\text { 2014, Reino Unido) }\end{array}$ \\
\hline We See it & $\begin{array}{c}\text { Amazon Prime } \\
\text { Vídeo }\end{array}$ & $2022-$ & Versión espacial & $\begin{array}{c}\text { Versión Serie Al } \\
\text { Haspectrum (2018, } \\
\text { Israel) }\end{array}$ \\
\hline
\end{tabular}

Fuente: Elaboración propia

\subsection{7. $H B O M a x$}

HBO Max es un servicio de vídeo bajo demanda por suscripción propiedad de WarnerMedia como parte del conglomerado AT\&T. WarnerMedia cuenta con diversas subsidiarias entre las que se encuentran el canal generalista The CW, los canales de cable CNN y HBO y el estudio cinematográfico Warner Bros. Pictures. HBO Max se lanzó en Estados Unidos en 2020 y actualmente se encuentra en expansión global, sustituyendo a $\mathrm{HBO}$ en los mercados donde existía como servicio de vídeo bajo demanda. 
A pesar del escaso tiempo que lleva en funcionamiento, en las series originales de HBO Max se aprecia una presencia significativa del reciclaje de material audiovisual, específicamente de reboots, revivals y spin-off de contenido de otras divisiones del grupo WarnerMedia, como HBO, Warner Bros. Television y Warner Bros. Pictures.

Tabla 7. Reciclajes narrativos en la producción original de HBO Max en EE.UU.

\begin{tabular}{|c|c|c|c|c|}
\hline Título & Servicio & Producción & Tipo & Material previo \\
\hline $\begin{array}{c}\text { Head of the } \\
\text { Class }\end{array}$ & HBO Max & 2021 & Reboot & $\begin{array}{c}\text { Serie Head of the } \\
\text { Class (ABC: } 1986- \\
\text { 1991) }\end{array}$ \\
\hline Gossip Girl & HBO Max & $2021-$ & Reboot & $\begin{array}{c}\text { Serie Gossip Girl (The } \\
\text { CW: 2007-2012) }\end{array}$ \\
\hline $\begin{array}{c}\text { And Just Like } \\
\text { That... }\end{array}$ & HBO Max & $2021-$ & Revival & $\begin{array}{c}\text { Serie Sex and the City } \\
\text { (HBO: 1998-2004) }\end{array}$ \\
\hline Peacemaker & HBO Max & 2022 & Spin-off & $\begin{array}{c}\text { Película The Suicide } \\
\text { Squad (2021) }\end{array}$ \\
\hline
\end{tabular}

Fuente: Elaboración propia

\subsubsection{Apple TV+}

Apple TV+ es un servicio de vídeo bajo demanda por suscripción propiedad de Apple Inc. Lanzado en 2019, cuenta con una modalidad de suscripción, pero es accesible gratuitamente durante un año por los compradores de productos de Apple. Inicialmente AppleTV+ se centró en su propio contenido original, con un número de títulos adquiridos muy minoritario. Frente a la importancia de adaptaciones literarias en su catálogo, el número de títulos que toman como referentes materiales audiovisuales previos es muy limitado y no se detecta ninguna tendencia significativa.

Tabla 8. Reciclajes narrativos en la producción original de Apple TV+ en EE.UU.

\begin{tabular}{|c|c|c|c|c|}
\hline Título & Servicio & Producción & Tipo & Material previo \\
\hline Amazing Stories & Apple TV+ & 2020 & Continuación & $\begin{array}{c}\text { Serie Amazing Stories } \\
\text { (NBC: 1985-1987) }\end{array}$ \\
\hline Acapulco & Apple TV+ & $2021-$ & Versión temporal & $\begin{array}{c}\text { Película How to Be a } \\
\text { Latin Lover (2017) }\end{array}$ \\
\hline Suspicion & Apple TV+ & $2022-$ & Versión espacial & $\begin{array}{c}\text { Serie Kfulim (2015- } \\
\text { 2019, Israel) }\end{array}$ \\
\hline
\end{tabular}

Fuente: Elaboración propia 


\subsection{EL RECICLAJE EN LA PRODUCCIÓN ORIGINAL DE LOS SERVICIOS DE VÍDEO BAJO DEMANDA EN ESPAÑA}

\subsubsection{Los servicios de vídeo demanda nacionales: Movistart, Atresplayer Premium y Filmin}

Movistar+ es una plataforma de televisión de pago resultado de la fusión en 2015 de las plataformas Canal+ (satélite) y Movistar TV (IPTV), y actualmente propiedad de Telefónica. En 2019 lanzó un servicio de vídeo bajo demanda por suscripción, Movistar+ Lite. Desde 2017 ha mantenido una ambiciosa estrategia de producción de contenidos originales donde el reciclaje de materiales narrativos audiovisuales ha tenido una presencia pequeña. Sin embargo, destacan dos spin-offs de series de la televisión generalista (Velvet Colección y Merlí: Sapere aude) como parte de una estrategia de diversificación de su contenido.

Atresplayer Premium es un servicio de vídeo bajo demanda por suscripción del grupo de comunicación Atresmedia. Fue lanzado en 2019 como modalidad de suscripción de los servicios de vídeo bajo demanda con publicidad del grupo, como Atresplayer y Flooxer. Actualmente, es primera ventana de las series de la cadena generalista Antena 3 y también mantiene una activa producción de series originales. El reciclaje de materiales audiovisuales previos tiene una fuerte presencia en su producción original, tomando como referentes series de Antena 3 ( 5 títulos) y cine producido por Atresmedia ( 1 título).

El servicio de vídeo bajo demanda por suscripción Filmin solo ha estrenado una serie original hasta este momento, que como adaptación literaria no cumple los requisitos para ser incorporada a la base de datos.

Tabla 9. Reciclajes narrativos en los servicios de VOD nacionales

\begin{tabular}{|c|c|c|c|c|}
\hline Título & Servicio & Producción & Tipo & Material previo \\
\hline Velvet Colección & Movistar+ & $2017-2019$ & Spin-off & $\begin{array}{c}\text { Serie Velvet (Antena } \\
\text { 3: 2014-2016) }\end{array}$ \\
\hline Skam España & Movistar+ & $2018-2020$ & Versión espacial & $\begin{array}{c}\text { Serie Skam (2015- } \\
\text { 2017, Noruega) }\end{array}$ \\
\hline El nudo & $\begin{array}{c}\text { Atresplayer } \\
\text { Premium }\end{array}$ & 2019 & Versión espacial & $\begin{array}{c}\text { Serie Amar después } \\
\text { de amar (2017, } \\
\text { Argentina) }\end{array}$ \\
\hline $\begin{array}{c}\text { Merlí: Sapere } \\
\text { aude }\end{array}$ & Movistar+ & $2019-2020$ & Spinoff & $\begin{array}{c}\text { Serie Merlí (TV3: } \\
2015-2018)\end{array}$ \\
\hline
\end{tabular}




\begin{tabular}{|c|c|c|c|c|}
\hline Título & Servicio & Producción & Tipo & Material previo \\
\hline $\begin{array}{l}\text { Física o química: } \\
\text { El reencuentro }\end{array}$ & $\begin{array}{l}\text { Atresplayer } \\
\text { Premium }\end{array}$ & 2020 & Revival & $\begin{array}{c}\text { Serie Física o química } \\
\text { (Antena 3: 2008- } \\
\text { 2011) }\end{array}$ \\
\hline \#Luimelia '77 & $\begin{array}{l}\text { Atresplayer } \\
\text { Premium }\end{array}$ & 2020 & Continuación & $\begin{array}{c}\text { Serie Amar en } \\
\text { tiempos revueltos } \\
\text { (Antena 3: 2013-) }\end{array}$ \\
\hline Mentiras & $\begin{array}{c}\text { Atresplayer } \\
\text { Premium }\end{array}$ & 2020 & Versión espacial & $\begin{array}{c}\text { Serie Liar (2017- } \\
\text { 2020, Reno Unido) }\end{array}$ \\
\hline \#Luimelia & $\begin{array}{l}\text { Atresplayer } \\
\text { Premium }\end{array}$ & $2020-2021$ & Spin-off & $\begin{array}{c}\text { Serie Amar en } \\
\text { tiempos revueltos } \\
\text { (Antena 3: 2013-) }\end{array}$ \\
\hline$A l b a$ & $\begin{array}{l}\text { Atresplayer } \\
\text { Premium }\end{array}$ & 2021 & Versión espacial & $\begin{array}{c}\text { Serie Fatmagül'ün } \\
\text { Suçu Ne? (2010-2012, } \\
\text { Turquía) }\end{array}$ \\
\hline $\begin{array}{c}\text { Los hombres de } \\
\text { Paco }\end{array}$ & $\begin{array}{l}\text { Atresplayer } \\
\text { Premium }\end{array}$ & 2021- & Revival & $\begin{array}{c}\text { Serie Los Hombres de } \\
\text { Paco (Antena 3: } \\
\text { 2005-2010) }\end{array}$ \\
\hline $\begin{array}{l}\text { Los protegidos: } \\
\text { El regreso }\end{array}$ & $\begin{array}{l}\text { Atresplayer } \\
\text { Premium }\end{array}$ & 2021- & Revival & $\begin{array}{c}\text { Serie Los protegidos } \\
\text { (Antena 3: 2010- } \\
\text { 2012) }\end{array}$ \\
\hline $\begin{array}{l}\text { Señor, dame } \\
\text { paciencia }\end{array}$ & $\begin{array}{l}\text { Atresplayer } \\
\text { Premium }\end{array}$ & 2022 & Versión temporal & $\begin{array}{l}\text { Película Señor, dame } \\
\text { paciencia (2017) }\end{array}$ \\
\hline
\end{tabular}

Fuente: Elaboración propia

4.2.2. Los servicios de vídeo demanda transnacionales: Netflix, Amazon Vídeo, $H B O$, Disney+ y Starz

Los principales servicios de vídeo bajo demanda por suscripción de Estados Unidos han ido llegando al mercado española de forma gradual, Netflix en 2015, Amazon Prime Vídeo en 2016, Apple TV+ en 2019, y Disney+ en 2020. Además, cadenas de televisión de pago como HBO (en 2016) y Starz (en 2020) han entrado en el mercado español con servicios de vídeo bajo demanda, aunque en el primer caso fue sustituido por HBO Max en 2021. Prácticamente todos estos grupos han iniciado estrategias de producción de contenido original en España, con diferente grado de intensidad. En relación al reciclaje de materiales narrativos audiovisuales, se trata de una modalidad con cierta presencia. Netfllix lo ha utilizado para crear contenido basado en sus propias series, mientras que Amazon Prime Vídeo ha tomado como referente series españolas que estaban en su catálogo. En el caso de HBO España, no se aprecia ninguna tendencia.

RAE-IC, Revista de la Asociación Española de Investigación de la Comunicación vol. 9, núm. 17 (2022), 25-50 
Tabla 10. Reciclajes narrativos en los servicios de VOD transnacionales en España

\begin{tabular}{|c|c|c|c|c|}
\hline Título & Servicio & Producción & Tipo & Material previo \\
\hline Criminal España & Netflix & 2019 & Continuación & $\begin{array}{c}\text { Serie Criminal UK } \\
\text { (2019-2020, Reino } \\
\text { Unido) }\end{array}$ \\
\hline Por Ho por B & HBO & 2020 & Versión temporal & $\begin{array}{c}\text { Cortometraje Pipas } \\
\text { (2013) }\end{array}$ \\
\hline $\begin{array}{c}\text { Élite: historias } \\
\text { breves }\end{array}$ & Netflix & $2021-$ & Continuación & $\begin{array}{c}\text { Serie Élite (Netflix: } \\
\text { 2018-) }\end{array}$ \\
\hline $\begin{array}{c}\text { El internado: Las } \\
\text { cumbres }\end{array}$ & Amazon Vídeo & $2021-$ & Reboot & $\begin{array}{c}\text { Serie El internado } \\
\text { (Antena 3: 2007- } \\
\text { 2010) }\end{array}$ \\
\hline $\begin{array}{c}\text { Historias para } \\
\text { no dormir }\end{array}$ & Amazon Vídeo & $2021-$ & Versión temporal & $\begin{array}{c}\text { Serie Historias para } \\
\text { no dormir (TVE1: } \\
\text { 1964-1968, 1982) }\end{array}$ \\
\hline $\begin{array}{c}\text { Sin novedad } \\
\text { HBO España }\end{array}$ & $2021-$ & Versión espacial & $\begin{array}{c}\text { Serie No Activity } \\
\text { (2015-2018. } \\
\text { Australia) }\end{array}$ \\
\hline
\end{tabular}

Fuente: Elaboración propia

\section{CONCLUSIONES}

El análisis de las series de ficción que se basan en el reciclaje de materiales audiovisuales previos muestra que es una tendencia en alza en los procesos de producción original de los servicios de vídeo bajo demanda en Estados Unidos. La relevancia de la continuación (20 títulos) y el spin-off (9 títulos) ejemplifican la importancia de las franquicias en el audiovisual contemporáneo, combinadas con la nostalgia en aquellas relacionadas con franquicias clásicas como Star Wars y Star Trek. La importancia de las versiones temporales (11 títulos), los revivals ( 6 títulos) y los reboots ( 3 títulos) también evidencian la importancia del capital nostálgico.

Cabe destacar aquí que, aunque Netflix y Amazon Prime Vídeo son los servicios más longevos y más activos en la producción, solo uno de ellos, Netflix, lo ha explorado de forma significativa. Desde el primer momento parte de la estrategia de producción de Netflix se basó sobre adaptaciones: su primera serie original, House of Cards (20132018), era versión de un referente británico (House of Cards, BBC1: 1990). Esto se puede relacionar con el interés de Netflix de conectar su producción original con contenido adquirido (presente a través de diferentes fórmulas), pero también con el rol de la nostalgia en su identidad de marca. 
La metáfora de la biblioteca, o de un catálogo, que contiene una colección de textos audiovisuales, resulta especialmente eficaz para describir la dinámica interna de Netflix. Cuando el recuerdo de una película o un programa de televisión no sólo se almacena en un inventario, sino que también se indexa y se pone a disposición de la búsqueda, la nostalgia mediada puede convertirse en una propiedad intrínseca para la sostenibilidad del archivo de Netflix y su modelo de negocio, de múltiples maneras (Taurino, 2019, pp. 10-11. Traducción propia).

Además, se constata el crecimiento del reciclaje de material narrativo como fórmula de producción en los últimos años: solo una cuarta parte de los títulos se estrenaron entre 2013 y 2017, mientras que las otras tres cuartas partes son posteriores. El año 2021, el último completo del estudio, muestra un boom atribuible sobre todo a Disney+, cuyos originales, salvo excepciones, se articulan sobre fórmulas reciclaje en consonancia por su tradicional apuesta por la explotación intensiva de su propiedad intelectual y la nostalgia (Gillan, 2019).

Se constata así el peso cuantitativo de las fórmulas de reciclaje de material audiovisual en los servicios de vídeo bajo demanda de los grupos de comunicación tradicionales (legacy media), como es el caso de Disney+, HBO Max, Peacock y Paramount+ en Estados Unidos y de Atresplayer Premium en España, como resultado de su acceso más directo a propiedad intelectual de eficacia ya probada. No siempre eso tiene la intencionalidad de aprovechar un capital nostálgico (como es el caso de la franquicia Marvel en Disney+), pero se trata de un factor relevante.

Por último, el marco comparativo pone de manifiesto que la producción local se adapta a las circunstancias de cada país. En España Amazon Prime Vídeo cuenta en su catálogo con numerosas series españolas de la televisión pública y privada, lo que se puede relacionar con que dos de sus originales las tomen como referencia. Sin embargo, esto no parece haber sido un factor para su producción en Estados Unidos. Para Netflix los referentes son sus propias series, en un marco de retroalimentación de contenido.

Como se pone de manifiesto en el análisis, el reciclaje de materiales audiovisuales es una tendencia de producción original de series significativa en los servicios de vídeo bajo demanda. Su estudio permite tanto examinar las estrategias que relacionan el contenido 
original con el adquirido como valorar elementos como la sinergia empresarial, la identidad de marca y la relevancia de la nostalgia en la cultura contemporánea.

\section{REFERENCIAS BIBLIOGRÁFICAS}

Ball, M. (27 de agosto de 2018). How the Paradox of the Term 'Original Series' Explains the Video Industry (Netflix Misunderstandings, Pt. 4). MatthewBall.vc. Recuperado de https://www.matthewball.vc/all/netflixoriginals

Bellamy, R. V., McDonald, D. G. y Walker, J. R. (1990). The spin-off as television program form and strategy. Journal of Broadcasting \& Electronic Media, 34(3), 283297. https://doi.org/10.1080/08838159009386743

Bisson, G. (2019). The Ampere Analysis mornings - Global drama trends: what's hot, where and why? Presentación en el Festival Série Series 2019. Recuperado de https://vimeo.com/349050288

Cascajosa Virino, C. (2006). El espejo deformado: versiones, secuelas y adaptaciones en Hollywood. Sevilla: Servicio de Publicaciones de la Universidad de Sevilla.

Cascajosa Virino, Concepción (2016). La cultura de las series. Barcelona: Laertes.

Castro, D, Rigby, J., Cabral, D. y Nisi, V. (2019). The binge-watcher's journey:

Investigating motivations, contexts, and affective states surrounding Netflix viewing. Convergence: The International Journal of Research into New Media Technologies, 27(1), p. 3-20. https://doi.org/10.1177/1354856519890856

Cuelenaere, E., Willems, G. y Joye, S. (Eds.) (2021). European Film Remakes. Edimburgo: University Press.

Davenport, T. y Beck, J. (2001). The Attention Economy: Understanding the New Currency of Business. Cambridge: Harvard Business School Press.

Dziadul, C. (1 de julio de 2021). Netflix takes lead in European scripted content. Broadband and TV News. Recuperado de https://cutt.ly/OPUwQZU 
Fernández, J. (31 de julio de 2021). ATRESplayer PREMIUM crece un 73\% y duplica los datos de Mitele PLUS en el primer semestre de 2021. Bluper. Recuperado de https://cutt.ly/xPUwE9X

Fernández Labayen, M. y Martín Morán, A. (2019). Manufacturing proximity through film remakes: Remake rights representatives and the case of local-language comedy remakes. Communications, 44(3), 282-303. https://doi.org/10.1515/commun-20192058

Fleury, J., Hartzheim, B. H. y Mamber, S. (2019). Introduction. The franchise era. En J. Fleury, B. H. Hartzheim y S. Mamber (Eds.), The Franchise Era: Managing Media in the Digital Economy (pp. 1-28). Edimburgo: Edinburgh University Press.

García Leiva, M.T. y Albornoz, L.A. (2021) VOD service providers and regulation in the European Union: An audiovisual diversity approach. International Journal of Cultural Policy, 27(3), 267-281. https://doi.org/10.1080/10286632.2020.1769614

Gil, Steven (2014), A Remake by Any Other Name: Use of a Premise Under a New Title. En C. Lavigne (Ed.), Remake Television: Reboot, Reuse, Recycle (pp. 21-36). Lanham: Lexington Books.

Gillan, J. (2019). TV Brand-casting, SVOD, and OTT at Comcast and Disney. En J. Fleury, B. H. Hartzheim y S. Mamber (Eds.), The Franchise Era: Managing Media in the Digital Economy (pp. 181-208). Edimburgo: Edinburgh University Press.

Henderson, S. (2017). The Hollywood Sequel: History \& Form, 1911-2010. Londres: Bloomsbury Publishing.

Herbert, D. (2018). Film Remakes and Franchises. New Brunswick: Rutgers University Press.

Iordache, C., Raats, T. y Afilipoaie, A. (2021). Transnationalisation revisited through the Netflix Original: An analysis of investment strategies in Europe. Convergence: The International Journal of Research into New Media Technologies, Online First. https://doi.org/10.1177/13548565211047344 
Jenner, M. (2018). Netflix and the Re-invention of Television. Londres: Palgrave Macmillan.

Jess-Cooke, C. (2009). Film Sequels: Theory and Practice from Hollywood to Bollywood. Edimburgo: Edinburgh University Press.

Johnson, D. (2013). Media Franchising: Creative License and Collaboration in the Culture Industries. Nueva York: New York University Press.

Lavigne, C. (2014). Introduction. En C. Lavigne (Ed.), Remake Television: Reboot, Reuse, Recycle (pp. 1-4). Lanham: Lexington Books.

Leitch, T. (2008). Adaptation Studies at a Crossroads. Adaptation, 1(1), 63-77. https://doi.org/10.1093/adaptation/apm005

Lobato, R. (2019). Netflix Nations: The Geography of Digital Distribution. Nueva York: NYU Press.

Lobato, R. y Lotz, A. (2021). Beyond Streaming Wars: Rethinking Competition in Video Services. Media Industries, 8(1), 89-108. https://doi.org/10.3998/mij.1338

Loock, K. (2018). American TV series revivals: Introduction. Television \& New Media, 19(4), 299-309. https://doi.org/10.1177/1527476417742971

Lotz, A. (2017). Portals: A Treatise on Internet-Distributed Television. Ann Arbor: Michigan Publishing.

Lotz, A. (2021). Media Disrupted: Surviving Pirates, Cannibals, and Streaming Wars. Cambridge: The MIT Press.

Low, E. (20 de abril de 2021). Netflix Reveals \$17 Billion in Content Spending in Fiscal 2021. Variety. Recuperado de https://cutt.ly/xPUwUWO

Mastrini, G. y Krakowiak, F. (2021). Netflix en Argentina: expansión acelerada y producción local escasa. Comunicación y Sociedad, 18, 1-23. https://doi.org/10.32870/cys.v2021.7915 
Mateos-Pérez, J. y Sirera-Blanco, R. (2021). Taxonomía de las series de televisión españolas en la era digital (2000-2020). El Profesional de la información, 30(6), 1-15. https://doi.org/10.3145/epi.2021.nov.08

Morillo, V. (29 de diciembre de 2021). La producción de series se mantiene en España durante 2021 a pesar de la pandemia. El Español. Recuperado de https://cutt.ly/KPUwLZp

Neira, E. (2020). Streaming Wars: La nueva televisión. Barcelona: Planeta.

Nicolaou, A. y Grimes, C. (29 de diciembre de 2021). Streaming wars drive media groups to spend more than $\$ 100$ bn on new content. Financial Times. Recuperado de https://cutt.ly/7PUw1pl

Novak, A. N. (2016). Framing the Future of Media Regulation through Netflix. En D. Smith-Rowsey (Ed.), The Netflix Effect. Technology and Entertainment in the 21st Century (pp. 33-48). Londres: Bloomsbury.

Pallister, K. (2019). Introduction. En K. Pallister (Ed.), Netflix Nostalgia: Streaming the Past on Demand (pp. 1-7). Lanham: Lexington Books.

Palomba, A. (2022). Building OTT brand loyalty and brand equity: Impact of original series on OTT services. Telematics and Informatics, 66, Online.

https://doi.org/10.1016/j.tele.2021.101733

Perkins, C. y Verevis, C. (Eds.) (2019). Transnational Television Remakes. Londres: Routledge.

Rosewarne, L. (2020). Why We Remake: The Politics, Economics and Emotions of Film and TV Remakes. Londres: Routledge.

Sanson, K. y Steirer, G. (2019). Hulu, streaming, and the contemporary television ecosystem. Media, Culture \& Society, 41(8), 1210-1227. https://doi.org/10.1177/0163443718823144 
Scarlata, A., Lobato, R. y Cunningham, S. (2021). Producing local content in international waters: the case of Netflix's Tidelands. Continuum: Journal of Media \& Cultural Studies, 35(1), 137-150. https://doi.org/10.1080/10304312.2021.1884654

Schneider, M. (7 de agosto de 2015). FX Boss: 'There Is Simply Too Much Television'. TV Insider. Recuperado de https://cutt.ly/sPUw7Te

Smith, I. R. y Verevis, C. (Ed.) (2017). Transnational Film Remakes. Edimburgo: Edinburgh University Press.

Spangler, T. (20 de enero de 2022). Netflix Falls Short of Q4 Subscriber Target, Stock Tumbles on Weak Forecast. Variety. Recuperado de https://cutt.ly/xPUw2mx Taurino, G. (2019). Crossing Eras. Exploring Nostalgic Reconfigurations in Media Franchises. En K. Pallister (Ed.), Netflix Nostalgia: Streaming the Past on Demand (pp. 9-24). Lanham: Lexington Books.

Thompson, D. (2017). Hit Makers: How Things Become Popular. Londres: Penguin UK. Verevis, C. (2006). Film Remakes. Edimburgo: Edinburgh University Press.

Villarejo, A. (2016). Jewish, Queer-ish, Trans, and Completely Revolutionary: Jill Soloway's Transparent and the New Television. Film Quarterly, 69(4), 10-22. https://doi.org/10.1525/fq.2016.69.4.10

Wayne, M. L. y Uribe Sandoval, A. C. (2021). Netflix original series, global audiences and discourses of streaming success. Critical Studies in Television: The International Journal of Television Studies, Online First. https://doi.org/10.1177/17496020211037259 White, P. (17 de febrero de 2022). FX's John Landgraf On Binge Drops Vs. Weekly Airings \& Being "Woefully Off" On When Peak TV Would Occur. Deadline. Recuperado de https://cutt.ly/PPUw8pa 